\title{
Influencia de las infiltraciones en la rehabilitación energética de la envolvente. El caso del plan de actuaciones en el parque público residencial de Andalucía
}

\section{Influence of infiltrations in the energy retrofit of the envelope. A case study from the action plans in the public housing stock of Andalusia}

\author{
C. E. Rodríguez-Jiménez ${ }^{(*)}$, M. J. Carretero-Ayuso ${ }^{(*)}$, J. C. Claro-Ponce $^{(*)}$
}

\section{RESUMEN}

El presente estudio presenta la investigación sobre la rehabilitación energética de la envolvente en una muestra de 615 viviendas sociales, incluidas dentro de los planes de construcción sostenible para el parque público residencial de Andalucía. Se evalúa de forma novedosa la relación entre las tasas de renovación de aire exterior y la demanda de climatización de viviendas en clima cálido. Ambas magnitudes ofrecen un paralelismo en los resultados, con valores promedio de 21,45\% de disminución de tasa de renovación y un 21,82\% de disminución en la demanda. Para ello, durante la ejecución de las intervenciones se ha desarrollado un muestreo de infiltraciones en viviendas. Las permeabilidades medidas se utilizan como parámetros de entrada en un proceso de modelizaciones energéticas bajo diferentes hipótesis, diferenciando la entrada de aire controlada o necesaria (ventilación) y la incontrolada o innecesaria (infiltraciones). Los resultados permiten visualizar el comportamiento de los edificios rehabilitados, cuantificando el ahorro energético como consecuencia de las infiltraciones para cada caso.

Palabras clave: Rehabilitación energética; infiltraciones de aire; tasas de renovación; test Blower Door.

\section{ABSTRACT}

The present study presents the research on the energy retrofit of the building envelope of 615 social housing that are part of the sustainable construction plans for the public housing stock of Andalusia (southern Spain). The analysis provides a new assessment of the relationship between the air-tightness and the energy demand for air conditioning of dwellings in warm climate. Both magnitudes offer a parallelism in the results, with average values of $21.45 \%$ decrease in air changes rate and $21.82 \%$ decrease in demand. To this end, during the implementation of interventions, tests of infiltrations have been developed in dwellings. The measured air permeability are used as input in a process of energy modeling under different approaches, differentiating the controlled air leakages (ventilation) and the uncontrolled or unnecessary ones (infiltration). The results show the behavior of buildings after refurbishment and energy savings by air leakage can be quantified in each case.

Keywords: Energy retrofit; air-tightness; air changes per hour; Blower Door test.

(*) Departamento de Construcciones Arquitectónicas II. Universidad de Sevilla. Sevilla (España).

${ }^{(* *)}$ Departamento de Expresión Gráfica. Universidad de Extremadura. Cáceres (España).

Persona de contacto/Corresponding author: ceugenio@us.es (C. E. Rodríguez-Jiménez)

ORCID: http://orcid.org/oooo-0oo3-3792-3389 (C. E. Rodríguez-Jiménez); http://orcid.org/oooo-0002-8082-8996

(M. J. Carretero-Ayuso); http://orcid.org/oooo-0oo1-8386-6958 (J. C. Claro-Ponce)

Cómo citar este artículo/Citation: C. E. Rodríguez-Jiménez, M. J. Carretero-Ayuso, J. C. Claro-Ponce (2018). Influencia de las infiltraciones en la rehabilitación energética de la envolvente. El caso del plan de actuaciones en el parque público residencial de Andalucía. Informes de la Construcción, 70(551): e271. https://doi.org/10.3989/ic.60450

Copyright: (C) 2018 CSIC. Este es un artículo de acceso abierto distribuido bajo los términos de la licencia de uso y distribución Creative Commons Reconocimiento 4.0 Internacional (CC BY 4.0). 


\section{INTRODUCCIÓN}

En Europa, el contexto actual para el ahorro de energía en edificación lo marca la Directiva Europea de Eficiencia Energética en Edificios (EPBD, 2010/31/EU) (1). En su texto se establecen un conjunto de medidas y objetivos destinados a disminuir el consumo energético no renovable y en consecuencia la emisión de los gases de efecto invernadero de los edificios. La directiva detalla que el $40 \%$ del consumo de energía UE corresponde a los edificios, por lo que su contribución en el cumplimiento de los fines medioambientales y energéticos marcados es muy decisiva. Además se establece, entre otras, la obligación de que a final de 2020 todos los edificios nuevos sean de consumo de energía casi nulo (nZEB).

En línea con estos objetivos, las actuaciones de mejora en la envolvente tienen una relación directa con el consumo energético y el confort de uso de los edificios. Ferreira et al (2), en su investigación en Portugal, concluyen que la mejora de los elementos de la envolvente está siempre presente en los paquetes de coste óptimo.

Dentro de la relación de la envolvente con el consumo de energía, el presente trabajo estudia el caso de la rehabilitación energética llevada a cabo en un conjunto de viviendas sociales en el sur de España. Los objetivos se centran en avanzar sobre el comportamiento de la permeabilidad al aire y su influencia en la demanda energética de climatización, dentro de un entorno de clima cálido, a partir de mediciones de infiltraciones in situ y modelizaciones virtuales.

Las tasas de infiltración de aire procedente del exterior son determinantes para la factura energética de un edificio, siendo responsables, según Pinto et al (3), de un intervalo entre el $30 \%$ y el $40 \%$ del gasto total de calefacción y refrigeración. A este respecto también se pronuncian Bobadilla et al (4), quienes realizan un estudio sobre 185 viviendas construidas en Chile entre 2007 y 2010, cifrando entre el 20 y el $50 \%$ el porcentaje de la demanda de energía por climatización debida a las infiltraciones a través de la envolvente, cuyos consumos equivalentes están comprendidos entre 23 y $203 \mathrm{kWh} /$ $\mathrm{m}^{2}$ año.

Ciertamente la permeabilidad a través de la envolvente, o volumen de aire que penetra al interior, debe ser suficiente para satisfacer las necesidades de salubridad pero sin sobrepasar umbrales de consumo energético razonables. Esta dualidad es la que distingue la ventilación (aire que penetra controladamente) de la infiltración (aire no controlado), tal como exponen Meiss y Feijó (5). Se hace necesario, por tanto, cuantificar con precisión cuáles son sus magnitudes, especialmente en el caso de las infiltraciones cuyo carácter incontrolado las hace ocasionar situaciones lesivas. A partir de ese punto se debe valorar adecuadamente el estado de diagnóstico encontrado y actuar en consecuencia.

El consenso internacional sobre limitaciones en los valores de infiltración es escaso. En España, donde se desarrolla la presente investigación, las prescripciones normativas no recogen la infiltraciones de aire, por lo que los edificios deben limitarse a las exigencias mínimas de ventilación por salubridad definidas en el Código Técnico de la Edificación (CTE) (6). En Europa existen numerosas referencias prescriptivas para infiltraciones de la envolvente en relación con la eficiencia energética, con cifras que van desde el o,6 $\mathrm{ACH}_{50}$ (Air
Changes Hour, 50Pa) que establece el estándar Passivhaus y la legislación en Portugal, hasta el valor de 10,0 $\mathrm{ACH}_{50}$ fijado para el Reino Unido, pudiendo promediarse la exigencia en torno a 4,5 $\mathrm{ACH}_{50}$ (7). En USA, el International Energy Conservation Code de 2012 (8) establece unas exigencias de $5, \mathrm{O} \mathrm{ACH}_{50}$ para zonas climáticas cálidas y $3,0 \mathrm{ACH}_{50}$ para las frías. En cualquier caso, para contrastar con las referencias normativas, hay de tener presente que éstas suelen prescribirse para edificios de nueva planta con una caracterización muy diferente a las viviendas construidas. En el análisis de éstas últimas es necesario contemplar que en muchos casos no se contaba con normativa de condiciones térmicas o ahorro de energía en el momento en el que fueron proyectadas.

Dentro de los elementos constructivos que conforman la envolvente, es destacable el importante papel que juegan las ventanas en relación a las infiltraciones de aire. El doble compromiso de ventilar sin penalizar la eficiencia energética debe ser una exigencia para estas unidades constructivas en el entorno actual. De ahí que su mejora o sustitución suela formar parte de las actuaciones de reforma en la envolvente con fines energéticos.

En efecto, las ventanas son responsables de una buena parte del volumen de aire permeable a través de la envolvente. Meiss y Feijó (9) desvelan que en torno al 50 \% de las infiltraciones pueden eliminarse sólo con la sustitución de ventanas, pasando de un valor inicial de 6,2 $\mathrm{ACH}_{50}$ a una media de 3,2 $\mathrm{ACH}_{50}$ tras la sustitución. Van den Bossche y Janssens (10) realizan un amplio estudio sobre permeabilidad de ventanas en Bélgica y afirman que éstas son un factor dominante para las infiltraciones de aire en los edificios ya que, incluso en situaciones de hermeticidad alta (con ventanas clase 4 según EN 12207:1999 (11) y $\mathrm{ACH}_{50}=1$ ), la media de impacto de la ventana en la permeabilidad total es del $14 \%$.

En la presente investigación se ha optado por centrar la permeabilidad mediante mediciones in situ, más allá de los valores de ensayo de ventanas en laboratorio, pues sólo así es posible verificar también las condiciones de colocación de la ventana en el hueco. La investigación realizada por d'Ambrosio et al (12) avala esta postura, ya que determinan altos valores de infiltración de aire en ventanas de altas prestaciones colocadas durante procesos de rehabilitación debidos a errores en la ejecución de obra.

La disminución de infiltraciones derivada de la mejora de ventanas se apunta como un factor clave para resolver el problema energético en rehabilitación de edificios. Además la sustitución de antiguas ventanas conlleva además una reducción de la transmitancia en la superficie del hueco por lo que resultan de gran eficacia en las intervenciones sobre la envolvente. Evangelisty et al (13) profundizan en este aspecto en su estudio sobre la rehabilitación energética en Roma donde dejan patente que el tipo de vidrio influye decisivamente en los resultados obtenidos. En los casos más extremos, la sustitución completa de ventanas obsoletas por otras con vidrios especiales de altas prestaciones, reducen la demanda en un 47\% para calefacción y el $40 \%$ en refrigeración.

\section{CASO DE ESTUDIO}

El estudio se lleva a cabo sobre 10 conjuntos residenciales ubicados en diferentes partes del territorio andaluz los cuales comprenden un total de 615 viviendas (tabla 1). Se trata de 
Tabla 1. Edificios que conforman la muestra seleccionada.

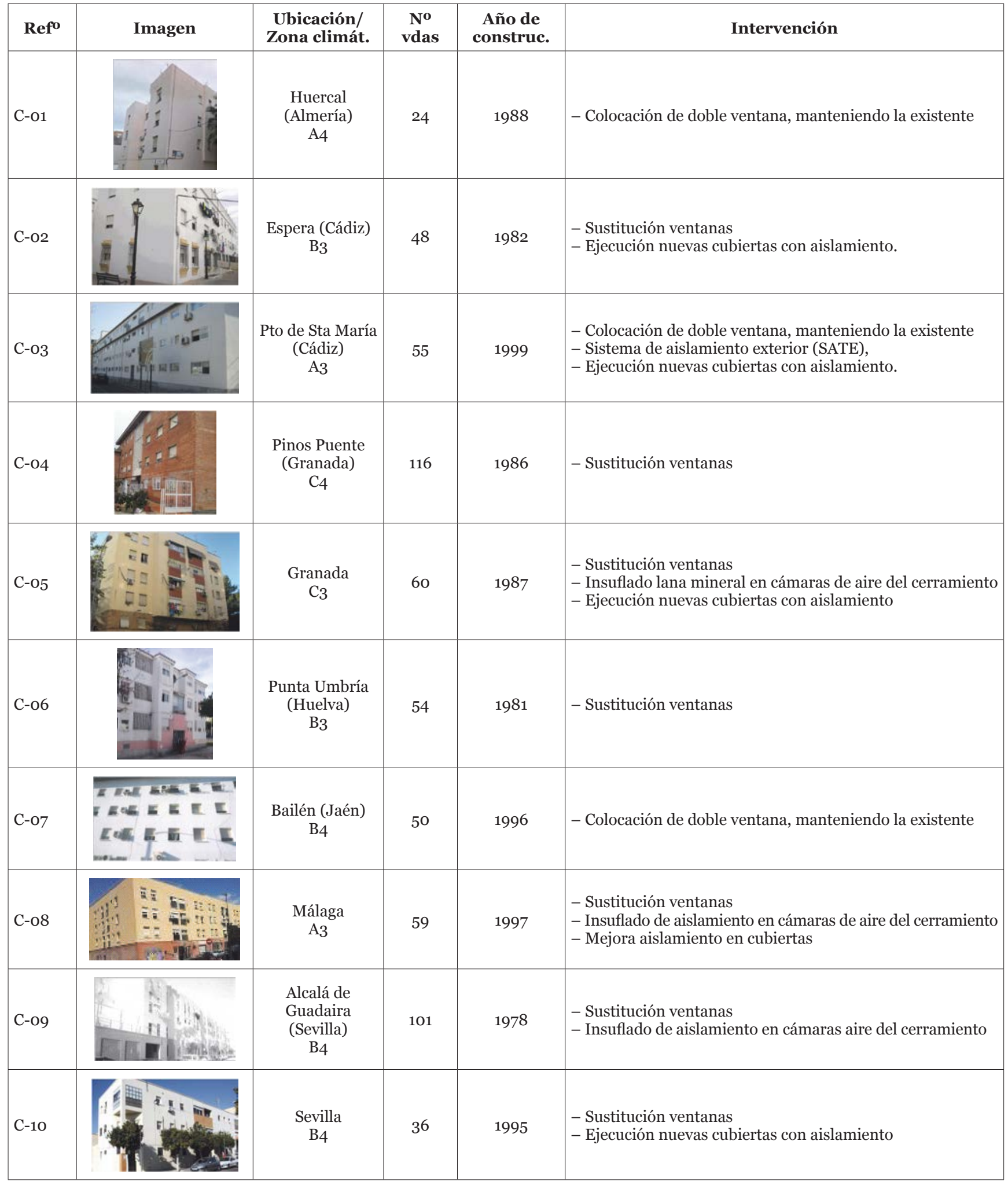

una muestra ubicada dentro de un programa de Rehabilitación Energética de viviendas sociales de titularidad pública gestionada por la Agencia de la Vivienda y Rehabilitación de Andalucía (AVRA), España.

La muestra comprende edificios plurifamiliares de viviendas sociales construidos principalmente en las dos últimas décadas del siglo XX. Geográficamente abarcan las principales zo- nas climáticas existentes en la región así como las tipologías constructivas predominantes en la zona.

La rehabilitación energética desarrollada sobre la envolvente es variable en función de la situación de partida de los edificios, incluyendo en todos los casos la mejora de huecos de ventanas, ya sea mediante sustitución o mediante colocación de doble ventana, conservando la existente. En la parte cie- 
ga se incorporan soluciones para incremento de aislamiento en cerramientos y cubiertas sólo en los casos en que éste es inexistente o muy escaso.

\section{METODOLOGÍA}

Se plantea un proceso metodológico basado en la obtención de datos de los edificios que conforman la muestra y la posterior modelización de los mismos bajo diversas hipótesis. Con ello se buscan resultados que permitan realizar un análisis y evaluación de las intervenciones de rehabilitación energética objeto de estudio.

\subsection{Obtención de parámetros}

La metodología se inicia con las labores para la definición constructiva de las intervenciones, creando un compendio ordenado de información de cara a conformar las simulaciones.

Esta recopilación abarca los proyectos técnicos redactados para cada una de las intervenciones donde existe en todos los casos un informe de inspección del estado de los edificios previo a la rehabilitación, realizado mediante inspecciones y determinaciones in situ (incluyendo medición de elementos, modificaciones introducidas, estado de conservación, etc.). Además se cuenta con toda la información obtenida del seguimiento en fase de obras, las cuales se ejecutan durante el periodo de investigación. Con ello, los datos de los elementos constructivos de la envolvente sobre los que se interviene son completos y precisos. La relación de las distintas unidades afectadas por la rehabilitación se refleja en la columna denominada "intervención" de la tabla 1.

De otra parte, las modelizaciones incorporan los resultados de los diferentes ensayos realizados durante el proceso. Los valores principales se obtienen de la medición de infiltraciones a través de ensayos de determinación de la estanqueidad al aire mediante presurización por medio de ventilador según lo descrito en la norma EN 13829:2000 (14), también conocido como Blower Door Test. Los ensayos se realizan tomando una vivienda representativa por cada edificio de la muestra, realizándose pruebas en dos fases clave del proceso: antes del inicio de las actuaciones y una vez concluidas las mismas Se pone así de manifiesto la variación de la estanqueidad al aire derivada de la rehabilitación.

Dentro de las opciones de comprobación de estanqueidad al aire contempladas en la citada norma, se aplica el Método B (ensayo de la envolvente del edificio). Siguiendo estas directrices, todas las aperturas del edificio al exterior dentro de la zona de ensayo (aberturas para salida de gases, aireadores, etc.) son cerradas herméticamente. Además en los paramentos que delimitan el área de la vivienda probada con otras dependencias interiores también se sellan todas las posibles vías de fugas de aire (puertas, rejillas, instalaciones, etc.). De tal modo el test determina la entrada de aire no controlada a través de la envolvente cuyo caudal viene dado por la expresión [1]

$$
V_{e n v}=C_{e n v} \cdot(\Delta P)^{n}
$$

Donde: $V_{e n v}$ es la tasa de flujo de aire a través de la envolvente em m $\mathrm{m}^{3} / \mathrm{h} ; C_{e n v}$ es el coeficiente del flujo de aire en $\mathrm{m}^{3} /\left(\mathrm{h} . \mathrm{Pa}^{\mathrm{n}}\right)$; $\Delta P$ es la diferencia de presión en Pa y $n$ es el exponente del flujo del aire.
Complementariamente a las pruebas Blower Door, se utiliza como técnica auxiliar la toma de imágenes mediante termografía infrarroja. Esta comprobación no tiene por objeto aportar valores para el diagnóstico o evaluación de los inmuebles, sino que se utiliza simplemente como control interno durante la realización de las citadas pruebas de permeabilidad al aire. Son imágenes que aportan información adicional sobre la localización de las infiltraciones, de forma que puedan detectarse y subsanarse desajustes puntuales en las unidades a probar (cierre de ventanas, filtraciones de otras dependencias interiores, etc.) las cuales podrían alterar los resultados. No obstante para la instrumentación se utilizan las pautas de la norma EN 13187:1998 (15).

\subsection{Modelizaciones}

Las modelizaciones informáticas se llevan a cabo con el empleo de la herramienta unificada LIDER-CALENER (HULC) versión 1.0.1558.1124 de diciembre 2016 que cuenta como Motor de Cálculo para simulación DOE-2.

Se trata de un programa reconocido en España por el Código Técnico de la Edificación español así como por el Ministerio de Fomento y el Ministerio de Industria, Energía y Turismo. A partir del esquema de certificación energética que esta herramienta plantea, se elaboran modelizaciones de cada uno de los edificios seleccionados en su estado inicial (previo a la intervención) así como es su estado final (tras la rehabilitación). El procedimiento seguido para confeccionar los modelos busca reproducir con rigor la realidad de los inmuebles y obtener fiabilidad en los resultados.

En este sentido, los datos de entrada al modelo relacionados con las especificaciones de rehabilitación derivan de la información obtenida en la fase anterior de la investigación, a partir de la documentación de proyecto y obra. Se definen así fielmente los parámetros constructivos y formales de las envolventes en ambos estados (inicial y final).

En cuanto a las tasas de renovación de aire, las diferentes hipótesis de simulación contemplan las dos fuentes de permeabilidad al aire (infiltración y ventilación). De tal modo, la magnitud que se introduce en cada modelo es la suma de dos cuantías:

- Por un lado se toma el valor real de infiltraciones a través de la envolvente (o entrada de aire incontrolada) medido in situ mediante las pruebas de estanqueidad al aire. En este punto hay que tener presente que la herramienta informática utilizada realiza las hipótesis de modelización a presión ambiente, fijada en 4Pa (5). Dado que los resultados del ensayo Blower door se definen a 50Pa de presión, es necesario trasladar los mismos a dicha presión ambiente. Para llevar a cabo esta conversión hay que considerar que, según la norma reguladora del test, EN 13829:2000 (14), las medidas de permeabilidades durante la prueba se determinan en un rango de presiones entre 10Pa y 10oPa, por lo que correlacionando ambas magnitudes se obtiene la función de la gráfica propia de cada caso. Por extrapolación, es posible determinar la tasa de infiltraciones de aire a $4 \mathrm{~Pa}$ que se introduce en el modelo.

- De otra parte, se añade la ventilación necesaria por salubridad, es decir, el aire controlado que aportan las instalaciones y rejillas existentes a tal efecto. Su volumen está fijado en el Documento Básico de Salubridad del Código Técnico de la Edificación (6). 


\section{RESULTADOS: ANÁLISIS Y DISCUSIÓN}

La tabla 2 recoge los resultados de renovaciones de aire a $50 P a$ obtenidas en los ensayos Blower door realizados en una vivienda representativa de cada edificio, antes y después de las intervenciones (estanqueidad al aire inicial y final, respectivamente). Se refleja también la disminución en la demanda de climatización debida a la rehabilitación, cuyo valor es la diferencia entre la cuantía que esta demanda arroja para el modelo en estado final menos su resultado paralelo en la simulación del estado inicial.

Tabla 2. Resultados Blower Door test y disminución de la demanda en los edificios objeto de estudio.

\begin{tabular}{|l|c|c|c|}
\hline Refo & $\begin{array}{c}\text { Estanqueidad } \\
\text { al aire inicial } \\
\text { (ACH }_{50} \text { ) }\end{array}$ & $\begin{array}{c}\text { Estanqueidad } \\
\text { al aire final } \\
\text { (ACH }_{50} \text { ) }\end{array}$ & $\begin{array}{c}\text { Disminución } \\
\text { de la demanda } \\
\mathbf{k W h} / \mathbf{m}^{2} \text { año }\end{array}$ \\
\hline C1 & 4.00 & 3.76 & 4.69 \\
\hline C2 & 11.44 & 2.51 & 24.78 \\
\hline C3 & 4.83 & 3.44 & 9.95 \\
\hline C4 & 12.85 & 3.09 & 44.50 \\
\hline C5 & 10.15 & 2.73 & 50.40 \\
\hline C6 & 13.46 & 3.45 & 27.10 \\
\hline C7 & 3.80 & 2.77 & 3.02 \\
\hline C8 & 6.22 & 3.26 & 6.61 \\
\hline C9 & 12.92 & 6.73 & 24.01 \\
\hline C10 & 4.08 & 2.96 & 9.68 \\
\hline
\end{tabular}

\subsection{Análisis de las tasas de renovación}

El análisis de resultados se inicia evaluando las tasas de renovación de aire obtenidas en los ensayos. Con el fin de contextualizar los resultados, se han recopilado resultados de diversos estudios de investigación publicados en la última dé- cada donde se determinan valores de hermeticidad $\left(\mathrm{ACH}_{50}\right)$ para viviendas en uso. Las pruebas realizadas sobre diversos grupos de muestreo ofrecen un abanico variable de resultados, en función de su ubicación geográfica y de las lógicas variaciones en las tipologías constructivas. De tal modo, en EEUU, el estudio publicado en 2013 por Chan el al (16), con una amplia base de datos de infiltraciones de aire que alcanza 134.000 viviendas unifamiliares, llega a una predicción media para el país de 12,o $\mathrm{ACH}_{50}$. Pero, por analogía, son los valores obtenidos en Europa los que mejor pueden ilustrar este aspecto. Las tablas 3 y 4 muestran los valores medios de otras investigaciones similares llevadas a cabo en el continente europeo, separadas por zona fría (norte) y cálida (sur).

Centrados en la zona cálida sur, donde se ubica el presente trabajo, la media de tasas de renovación de los estudios allí considerados es de 6,9 $\mathrm{ACH}_{50}$. Este valor comprende edificios de diferente antigüedad, tipología y sin rehabilitar, por lo que sería comparable con al valor medio inicial obtenido en este estudio, cifrado en $8,3 \mathrm{ACH}_{50}$. Este dato nos indica que los edificios objeto de la investigación están en situación más desfavorable, en cuanto a condiciones de permeabilidad al aire, que otros grupos residenciales afines del entorno geográfico próximo. Sin embargo al comparar los valores tras rehabilitar, el resultado medio del parque andaluz analizado $\left(3,5 \mathrm{ACH}_{50}\right)$ mejora el promedio final que se determina en el trabajo de Ramos et al $\left(6,8 \mathrm{ACH}_{50}\right)$, (25) y se sitúa en línea con los resultados obtenidos por Meiss y Feijó $\left(3,2 \mathrm{ACH}_{50}\right)(9)$.

Además del análisis de datos, en la valoración de estas intervenciones cabe considerar también la componente de mejora en el confort y habitabilidad para los usuarios. En este sentido, los estudios de Kurnitski (26) y Kalamees (18) coinciden en afirmar que valores de $\mathrm{ACH}_{50}$ superiores a $6 \mathrm{~h}^{-1}$, comunes para viviendas sin rehabilitar, provocan fluctuaciones de temperatura interiores que son percibidas por los ocupantes

Tabla 3. Valores de hermeticidad en estudios recientes realizados en países del norte de Europa.

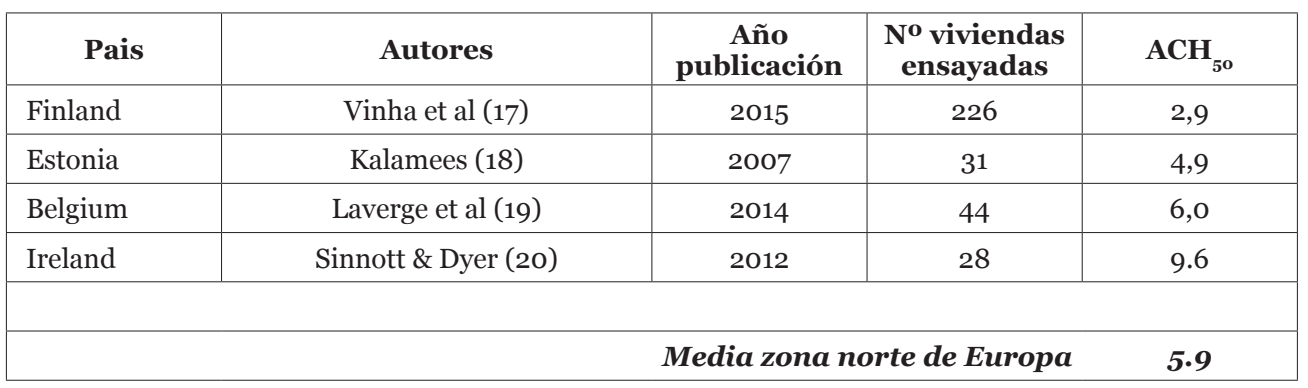

Tabla 4. Valores de hermeticidad en estudios recientes realizados en países del sur de Europa.

\begin{tabular}{|c|c|c|c|c|}
\hline Pais & Autores & $\begin{array}{c}\text { Año } \\
\text { publicación }\end{array}$ & $\begin{array}{l}N^{\circ} \text { viviendas } \\
\text { ensayadas }\end{array}$ & $\mathbf{A C H}_{50}$ \\
\hline Spain & Meiss/Feijó-Muñoz (21) & 2015 & 13 & 6,3 \\
\hline Spain & Fdez-Agüera et al (22) & 2016 & 45 & 5,7 \\
\hline Greece & Sfakianaki et al (23) & 2008 & 20 & 7,0 \\
\hline Italy & Alfano et al (24) & 2012 & 20 & 7,3 \\
\hline Portugal & Ramos et al (25) & 2015 & 25 & 8,9 \\
\hline Portugal & Pinto et al (3) & 2011 & 7 & 6,1 \\
\hline \multicolumn{4}{|c|}{ Media zona sur de Europa } & 6,9 \\
\hline
\end{tabular}


como desagradables y pueden hacer que algunas dependencias sean consideradas zonas poco confortables para las personas.

\subsection{Análisis energético}

El análisis energético aborda las repercusiones en la demanda de energía que la rehabilitación conlleva, con clara atención sobre las infiltraciones.

De acuerdo la metodología expuesta, se parte de un conjunto de ensayos de permeabilidad al aire realizados in situ así como de los modelos virtuales basados en las especificaciones constructivas y tasas de renovación de aire totales (las cuales son la suma de las infiltraciones reales medidas en los citados ensayos más la ventilación prescriptiva correspondiente).

En primera instancia, del contraste entre los resultados obtenidos para los estados inicial y final de los edificios se destaca la clara proporcionalidad existente entre la disminución porcentual de la demanda y la reducción en las tasas de renovación totales modelizadas, independientemente del grado de permeabilidad inicial de las viviendas.

La figura 1 representa gráficamente la citada comparativa, visualizándose el paralelismo entre ambas magnitudes para todos los edificios estudiados, lo que pone de manifiesto la alta repercusión de la hermeticidad en los ahorros energéticos obtenidos.

Este punto queda avalado también por el estudio realizado por d'Ambrosio et al (12) sobre mejora de ventanas en edificios en el área mediterránea, donde se alcanzan valores medios de $31,3 \%$ de ahorro en la demanda con un $37,4 \%$ de disminución en renovaciones de aire.

Para afrontar los datos de manera más específica, la siguiente fase del estudio desarrolla una categorización de los valores analizados, acotando la dispersión existente en los resultados de los test Blower Door. La justificación a esta amplitud en el rango de valores de infiltración hay que buscarla en las diferencias constructivas que presentan las ventanas, principales responsables de la estanqueidad al aire en la envolvente.

Por consiguiente, se divide la muestra analizada en dos bloques en función de las características de sus ventanas (tabla 5). El primer grupo recoge aquellas viviendas con ventanas más obsoletas, normalmente con perfilería de acero, y claras muestras de falta de ajustes en sus mecanismos de cierre (figura 2) donde los volúmenes de aire infiltrado son siempre elevados. La segunda agrupación incluye ventanas de diseño más avanzado, con perfilería de aluminio y elementos específicos de estanqueidad (figura 3), cuyas cifras de permeabilidad son más moderadas.

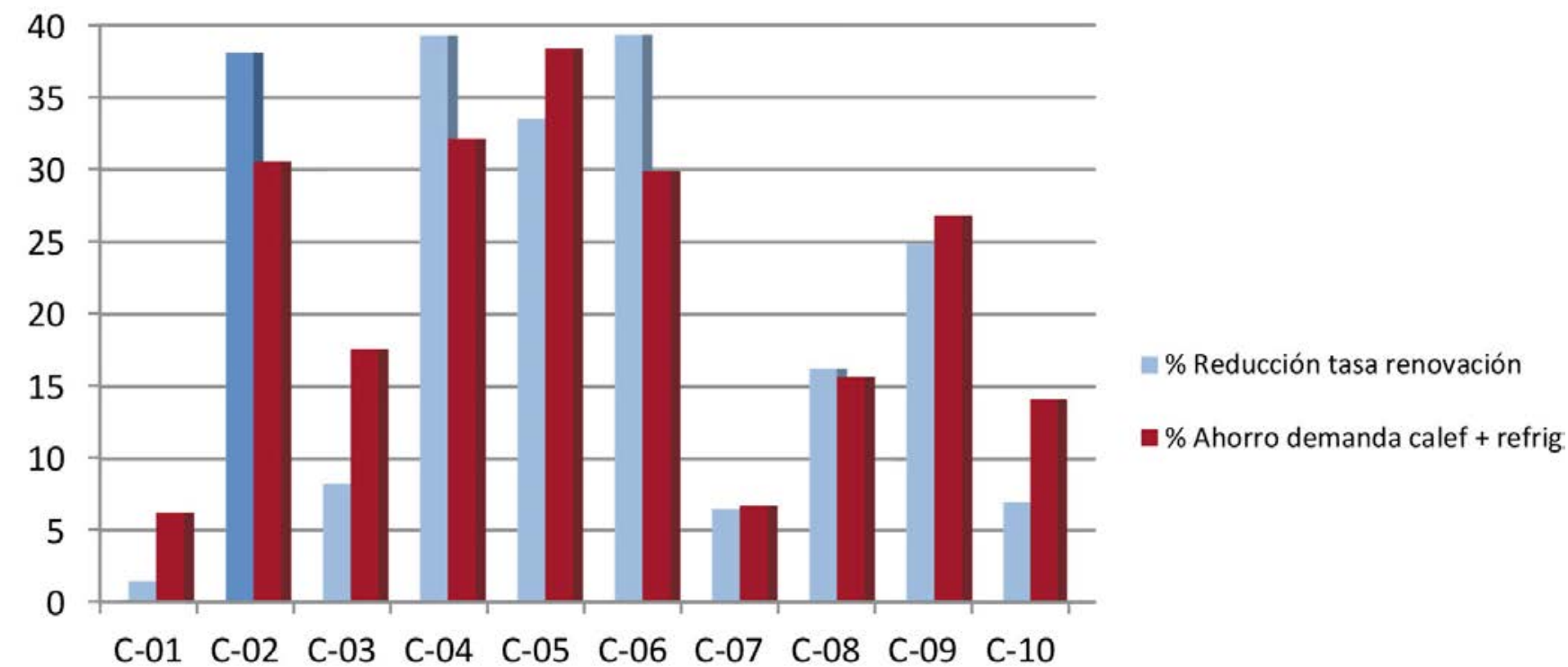

Figura 1. Contraste entre reducción de tasas de renovación totales (infiltraciones+ventilación) y ahorros de la demanda obtenidos.

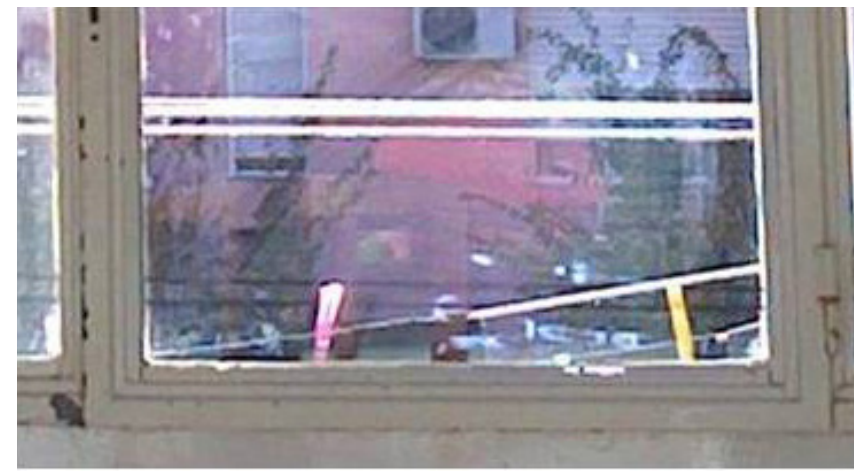

Figura 2. Ventana con perfiles de acero (C-09).

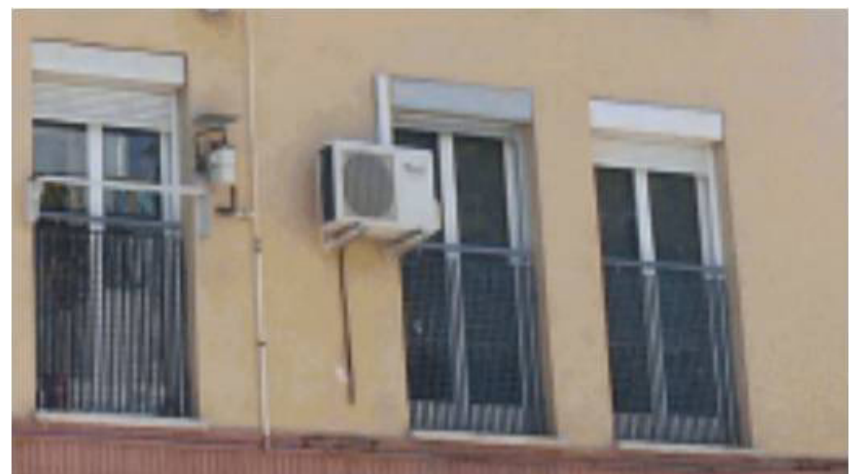

Figura 3. Ventana con perfiles de aluminio (C-o8). 
Tabla 5. Disminución de las tasas de renovación totales (infiltraciones+ventilación) y demandas en la muestra analizada.

\begin{tabular}{|c|c|c|c|}
\hline & $\operatorname{Ref}^{\circ}$ & $\begin{array}{c}\text { \% disminución } \\
\text { tasa renovación } \\
\text { de aire }\end{array}$ & $\begin{array}{c}\text { \% disminución } \\
\text { demanda conjunta }\end{array}$ \\
\hline \multirow{7}{*}{ 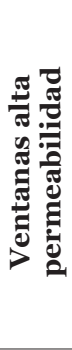 } & $\mathrm{C} 2$ & 38,11 & 30,57 \\
\hline & $\mathrm{C}_{4}$ & 38,29 & 32,13 \\
\hline & $\mathrm{C}_{5}$ & 33,52 & 38,39 \\
\hline & C6 & 39,32 & 29,88 \\
\hline & C9 & 24,85 & 26,85 \\
\hline & media & 35,02 & 31,56 \\
\hline & Desviación $\sigma$ & 6,00 & 4,27 \\
\hline \multirow{7}{*}{ 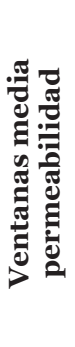 } & C1 & 1,47 & 6.25 \\
\hline & $\mathrm{C}_{3}$ & 8,25 & 17,62 \\
\hline & $\mathrm{C}_{7}$ & 6,52 & 6,74 \\
\hline & $\mathrm{C} 8$ & 16,23 & 15,67 \\
\hline & C10 & 6,96 & 14,11 \\
\hline & media & 7,89 & 12,08 \\
\hline & Desviación $\sigma$ & 5,33 & 4,75 \\
\hline
\end{tabular}

Tabla 6. Valores de ahorro en hipótesis sin infiltraciones (grupo de muestra con alta permeabilidad).

\begin{tabular}{|c|c|c|}
\hline Referencia & $\begin{array}{c}\text { Disminución } \\
\text { demanda conjunta } \\
\text { (calef+refrig) } \\
\text { kWh/m } \mathbf{2} \text { año }\end{array}$ & $\begin{array}{c}\text { Ahorro en } \\
\text { demanda conjunta } \\
\text { (calef+refrig) } \\
\text { \% }\end{array}$ \\
\hline $\mathrm{C} 2$ & 30,31 & 37,40 \\
\hline $\mathrm{C} 4$ & 62,42 & 45,07 \\
\hline $\mathrm{C} 5$ & 61,42 & 46,78 \\
\hline $\mathrm{C} 6$ & 41,45 & 45,69 \\
\hline $\mathrm{C} 9$ & 50,90 & 56,93 \\
\hline Media & $\mathbf{4 9 , 3 0}$ & $\mathbf{4 6 , 3 7 \%}$ \\
\hline
\end{tabular}

Una vez clasificada la muestra, se plantea una hipótesis para el estado final (tras rehabilitar) en la que ficticiamente desaparecen totalmente las infiltraciones no controladas, es decir, la permeabilidad al aire de toda la envolvente sería sólo la estrictamente necesaria por salubridad. En los modelos se reproduce introduciendo un valor de renovaciones de aire igual al caudal mínimo por ventilación que prescribe el Código Técnico español (6). Esta situación supone la menor entrada posible de aire exterior al edificio en condiciones de uso y por tanto marca el óptimo teórico de mejora que ofrecen las soluciones constructivas implementadas. El grupo de elevada permeabilidad ofrece resultados cuya magnitud permite visualizar mejor la modelización descrita (tabla 6).

Esta hipótesis fija la cota máxima de ahorro que podría alcanzar la demanda en lo que a infiltraciones de aire se refiere, lo cual es útil para contrastar los resultados de la situación real. La comparativa de la demanda obtenida sin infiltraciones frente a las demandas anterior y posterior a la rehabilitación es clarificadora (figura 4). Los edificios poseen margen de mejora aún después de las actuaciones implementadas, si bien su magnitud no es elevada, lo que revela que la rehabilitación ejecutada ha alcanzado cotas de ahorro notables.

Paralelamente se plantea también para el estado inicial la misma modelización sin infiltraciones, obteniéndose la evolución de la muestra al margen de estas entradas de aire, es decir, el ahorro que proporcionan por sí solas las soluciones de mejora de la transmitancia. Se trata de la contribución debida a las actuaciones sobre la parte opaca de la envolvente (incremento de aislamiento), así como la menor conductividad térmica de las nuevas ventanas.

De tal forma es posible desglosar la disminución de la demanda total en dos factores; el originado por la reducción de transmitancia térmica y el provocado por la merma de infiltraciones de aire.

Si consideramos los valores por grupos de muestra, la media del ahorro en la demanda conjunta refrigeración/calefacción

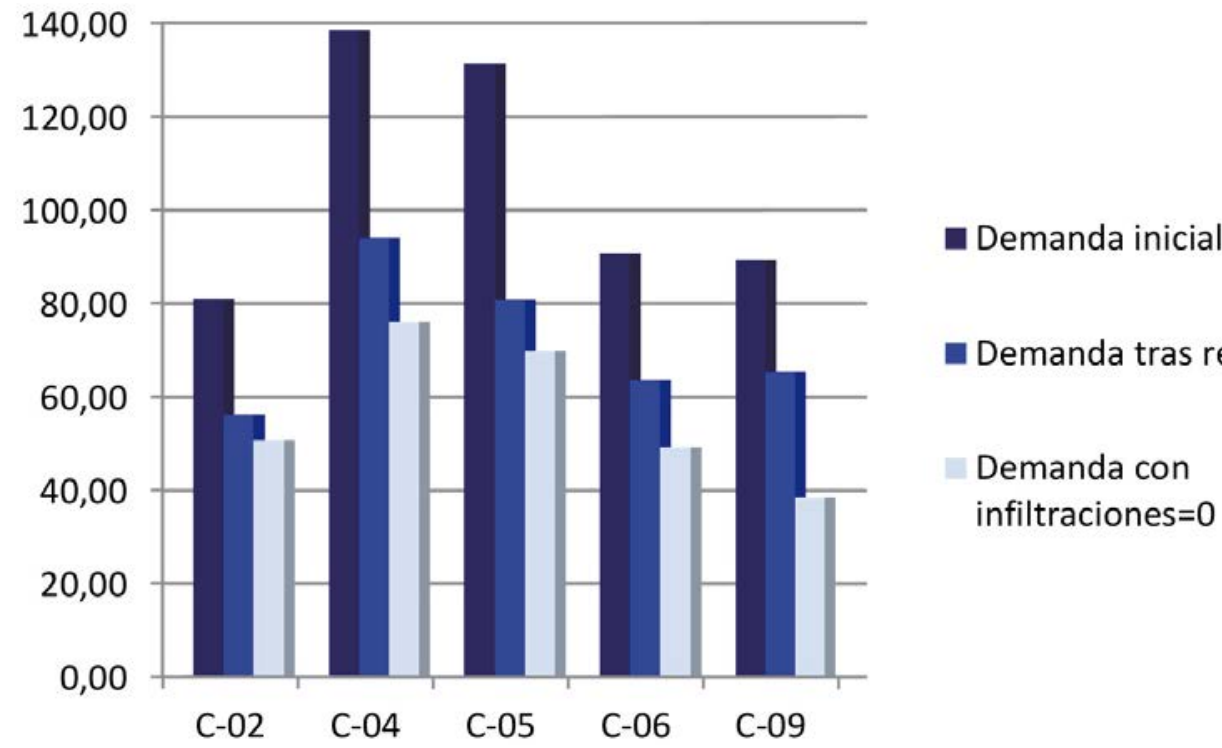

Figura 4. Comparativa de la demanda de climatización $\mathrm{kWh} / \mathrm{m} 2$ año en tres hipótesis consideradas. 
para los cinco edificios con mayor permeabilidad es del 34,16 $\mathrm{kWh} / \mathrm{m}^{2} \mathrm{año}$, dentro del cual la merma de infiltraciones determinan el grueso de esta mejora con $30,06 \mathrm{kWh} / \mathrm{m}^{2}$ año, mientras la disminución de la transmitancia repercute en los 4,10 kWh/ $\mathrm{m}^{2}$ año restantes. En el grupo de menor permeabilidad, con un ahorro medio es de $6,79 \mathrm{kWh} / \mathrm{m}^{2}$ año, las reducidas magnitudes de infiltraciones iniciales hacen que la reducción de las mismas consiga atenuar la demanda tan solo en $1,89 \mathrm{kWh} / \mathrm{m}^{2}$ año, mientras los efectos de la menor transmitancia son similares a los del grupo anterior, siendo responsables de $4,90 \mathrm{kWh} / \mathrm{m}^{2}$ año de ahorro.

Desde el punto de vista comparativo, estos resultados están en línea con diversas referencias publicadas para estudios realizados en España. Alonso et al (27) cifran el ahorro de energía por la incorporación de sistemas de aislamiento térmico exterior (ETICS) en el 15,40\%, mientras una solución similar del presente estudio (conjunto C-O3) aporta un ahorro del 13,97\%. De igual manera el contraste de los resultados obtenidos en el caso $\mathrm{C}_{5}$ con los datos publicados por Fernández-Agüera et al (28), (ambos casos recogen actuaciones similares en la envolvente), la comparativa arroja unos resultados muy próximos, ya que las pérdidas de infiltraciones dan lugar, respectivamente, a un $18,8 \%$ y un $17,0 \%$ de ahorro sobre la demanda inicial.

En cuanto al orden de magnitudes, se aprecia que los valores obtenidos como consecuencia de la mejora de aislamientos son discretos. Esta situación puede interpretarse, en primera instancia, dudando sobre la viabilidad de la intervención en la zona opaca de fachadas y cubiertas, sobre todo en los casos con infiltraciones de aire abundantes. Además este tipo de partidas suelen acaparar la mayor parte del presupuesto en la rehabilitación de la envolvente.

Sin embargo en este punto hay que ir más allá de las consideraciones energéticas y considerar que las reparaciones en la envolvente implican también renovación de la impermeabilización, subsanación de defectos superficiales (zonas degradadas, fisuraciones, roturas, etc.), de forma que mejora notablemente el comportamiento ante humedades interiores, condensaciones, acústica, estabilidad de revestimientos y otras lesiones frecuentes en edificios sin rehabilitar. Sin olvidar también los efectos estéticos cuyo beneficio es indudable para los ocupantes y su entorno. Giancola et al (29) ponen de manifiesto que estas intervenciones en viviendas sociales cubren la necesidad de alcanzar unos parámetros de habitabilidad razonables, además de evitar los riesgos de la llamada "pobreza energética" y sus derivadas sociales.

\section{CONCLUSIONES}

El presente estudio pone de manifiesto la elevada influencia de las infiltraciones en el comportamiento energético de los edificios residenciales. La mayor parte del ahorro alcanzado en la demanda conjunta de refrigeración y calefacción para la muestra estudiada se debe a la reducción de infiltraciones, con una sorprendente proporcionalidad entre ambas magnitudes cuando se comparan las disminuciones entre tasas de renovación totales y demanda.

Destaca asimismo el significativo descenso en los volúmenes de infiltración tras la rehabilitación. Las pruebas Blower door realizadas arrojan inicialmente unos valores de tasas de renovación con un promedio de $8,3 \mathrm{ACH}_{50}$, el cual es compara- tivamente alto frente a la media de 6,9 $\mathrm{ACH}_{50}$ obtenida para viviendas europeas en clima cálido. Sin embargo, tras la rehabilitación las cifras descienden sustancialmente por debajo de la mitad, con una media de $3,5 \mathrm{ACH}_{50^{\circ}}$

Consecuentemente las intervenciones consiguen valores de ahorro importantes y las cifras más reveladoras se obtienen en el grupo de inmuebles con mayor permeabilidad inicial, cuyo promedio de disminución de la demanda es el 31,56\%. En este mismo grupo se realiza una hipótesis ficticia que fija el máximo ahorro teórico por infiltraciones (lo que se consigue limitando las renovaciones al mínimo prescriptivo por salubridad), arrojando un valor medio de reducción en la demanda del 46,37\%. La comparativa entre este porcentaje máximo y el obtenido tras rehabilitar permite visualizar el margen de mejora que restaría aún por alcanzar. En cualquier caso, hay que tener presente que acercarse al óptimo planteado puede ser de difícil puesta en práctica para la muestra objeto de estudio, ya que las soluciones de las que se parte en las envolventes analizadas (con disposiciones constructivas elementales y bajas prestaciones energéticas) suelen requerir reformas de gran envergadura para adaptarlas a las exigencias actuales.

La alta repercusión de las infiltraciones sobre la demanda de climatización hace que, entre las soluciones implementadas, la incorporación de ventanas con baja permeabilidad al aire merezca una especial mención. Se trata de una de las partidas con menor peso en el presupuesto de la rehabilitación estudiada, lo cual implica que la intervención practicada sobre estos elementos ha logrado alcanzar valores significativos de ahorro en la factura energética con una inversión moderada. Este punto es de vital importancia en el diseño de planes de rehabilitación en viviendas, donde es fundamental un estudio previo que determine el estado de hermeticidad de las carpinterías, para actuar en consecuencia.

La investigación aborda también la influencia de las soluciones relacionadas con la mejora de la transmitancia de la envolvente (incremento de aislamientos en la parte opaca y la incorporación de ventanas con mayor resistencia térmica, principalmente), donde se alcanzan valores moderados de ahorro, en torno a $5,5 \mathrm{kWh} / \mathrm{m}^{2}$ año de media. No obstante para valorar de forma completa este tipo de actuaciones sobre las superficies ciegas, deben considerarse también otros aspectos que se infieren de las mismas, ya que implican también importantes aportaciones para los usuarios en clave de mejora de habitabilidad y confort así como un impacto positivo en la regeneración urbana y social.

\section{AGRADECIMIENTOS}

El presente trabajo está enmarcado dentro del acuerdo de Colaboración entre la Universidad de Sevilla y la Agencia para la Vivienda y Rehabilitación de Andalucía (AVRA), en materia de análisis y tratamiento de datos para mejora de eficiencia energética del parque público de viviendas. Todo ello al amparo del Decreto Ley 1/2014 de la Consejería de Fomento y Vivienda de la Junta de Andalucía que desarrolla el Programa de Impulso a la Construcción Sostenible en Andalucía, con la ayuda de fondos europeos FEDER (30).

Para la realización de las pruebas se ha contado con los recursos del Servicio de Control de Calidad de la citada Consejería así como el apoyo de la empresa Arquiges S.L.U. 


\section{REFERENCIAS}

(1) EPBD, Directive 2010/31/EU of the European Parliament and the Council of 19th May 2010 on Energy Performance of Buildings (recast), Official J. of Eur. Communities L153 (2010).

(2) Ferreira, M., Almeida, M., Rodrigues, A., Silva, S.M. (2016). Comparing cost-optimal and net-zero energy targets in building retrofit. Building Research and Information, 44 (2): 188-201, doi: 10.1080/09613218.2014.975412

(3) Pinto, M., Viegas, J., de Freitas, V.P. (2011). Air permeability measurements of dwellings and building components in Portugal. Building and Environment, 46 (12): 2480-2489, doi: 10.1016/j.buildenv.2011.06.009

(4) Bobadilla, A., Ossio, F., Figueroa, R., González, A., Muriel, D. C., \& Arriagada, R. (2014). The energy impact of envelope leakage. The Chilean Case. 35th AIVC Conference, 4th TightVent Conference and 2nd venticool Conference, Poznan, Poland

(5) Meiss, A., \& Feijó-Muñoz, J. (2013). La necesidad de estudiar las infiltraciones: estudio de caso en viviendas sociales de la urbanización Zabalgana (Vitoria). Boletín Académico, 3, 57-64.

(6) Ministerio de Fomento. España. Real Decreto 314/2006, por el que se aprueba el Código Técnico de la Edificación (CTE), de 17 de marzo. Boletín Oficial del Estado, 28 de marzo de 2006.

(7) Ossio, F., De Herde, A., Veas, L. (2012). Exigencias europeas para infiltraciones de aire: Lecciones para Chile. Revista de la construcción 11(1) 54-63.

(8) U.S. Department of Energy, Energy Efficiency and Renewable Energy, Building Technologies Program: Air Leakage Guide. Building Energy Codes (2011).

(9) Meiss, A., Feijó-Muñoz, J., Padilla-Marcos, M. A. (2016). Evaluación, diseño y propuestas de sistemas de ventilación en la rehabilitación de edificios residenciales españoles. Estudio de caso. Informes de la Construcción, 68(542): e148, doi: http://dx.doi.org/10.3989/ic.15.101.

(10) Van Den Bossche, N., Janssens, A. (2016). Airtightness and watertightness of window frames: Comparison of performance and requirements. Building and Environment, 110 (2016): 129-139, doi: 10.1016/j.buildenv.2016.09.034

(11) CEN (1999). EN 12207. Windows and doors. Air permeability. Classification. Brussels. European Committee for Standardization (CEN).

(12) D’Ambrosio Alfano, F.R., Dell'Isola, M., Ficco, G., Palella, B.I., Riccio, G. (2016). Experimental air-tightness analysis in mediterranean buildings after windows retrofit. Sustainability, 8(10), 991; doi: 10.3390/su8100991

(13) Evangelisti, L., Guattari, C., Gori, P. (2015). Energy retrofit strategies for residential building envelopes: An Italian case study of an early-50s building. Sustainability, 7(8): 10445-10460, doi: 10.3390/su70810445

(14) CEN. (2000). EN 13829. Thermal performance of buildings. Determination of air permeability of bulidings. Fan pressurization method. Brussels: European Committee for Standardization (CEN).

(15) CEN. (1998). EN 13187. Thermal performance of buildings. Qualitative detection of thermal irregularities in building envelopes. Infrared method. Brussels: European Committee for Standardization (CEN).

(16) Chan, W.R., Joh, J., Sherman, M.H. (2013). Analysis of air leakage measurements of US houses. Energy and Buildings, 66, 2013 : 616-625. doi: 10.1016/j.enbuild.2013.07.047

(17) Vinha, J. ,Manelius, E., Korpi, M., Salminen, K., Kurnitski, J., Kiviste, M., Laukkarinen, A. (2015). Airtightness of residential buildings in Finland. Building and Environment, 93 (2015): 128-140 doi: 10.1016/j.buildenv.2015.06.011

(18) Kalamees, T. (2007). Air tightness and air leakages of new lightweight single-family detached houses in Estonia. Building and Environment,42 (2007): 2369-2377 doi: 10.1016/j.buildenv.2006.06.001

(19) Laverge, J., Delghust, M., Van Den Bossche, N., Janssens, A. (2014). Airtightness assessment of single familiy houses in Belgium. International Journal of Ventilation, 12 (4): 379-389. doi: 10.1080/14733315.2014.11684031

(20) Sinnott D., Dyer M. (2012). Air-tightness field data for dwellings in Ireland. Building and Environment, 51 (2012): 269275. doi: 10.1016/j.buildenv.2011.11.016

(21) Meiss, A., Feijó-Muñoz, J. (2015) The energy impact of infiltration: a study on buildings located in north central Spain, Energy Efficiency 8 :51-64 doi: 10.1007/s12053-014-9270-x

(22) Fernández-Agüera, J.,Domínguez-Amarillo, S., Sendra, J.J., Suárez, R. (2016). An approach to modelling envelope airtightness in multi-family socialhousing in Mediterranean Europe based on the situation in Spain. Energy and Buildings 128 (2016): 236-253. http://dx.doi.org/10.1016/j.enbuild.2016.06.074

(23) Sfakianaki, A., Pavlou, K., Santamouris, M., Livada, I., Assimakopoulos, M. N., Mantas, P., et al. (2008). Air tightness measurements of residential houses in Athens, Greece. Building and Environment, 43: 398-405. doi: 10.1016/j.buildenv.2007.01.006

(24) Alfano, F., Dell’ Isola, M., Ficco, G., Tassini, F. (2012). Experimental analysis of air tightness in Mediterranean buildings using the fan pressurization method. Building and Environment, 53: 16-25. doi: 10.1016/j.buildenv.2011.12.017

(25) Ramos, N.M.M., Almeida, R.M.S.F., Curado, A., Pereira, P.F., Manuel, S., Maia, J. (2015). Airtightness and ventilation in a mild climate country rehabilitated social housing buildings - What users want and what they get. Building and Environment 92 (2015): 97-110 http://dx.doi.org/10.1016/j.buildenv.2015.04.016

(26) Kurnitski, J., Eskola, J., Palonen, J., Seppänen, O. (2005). Ventilation in 102 Finnish single-family houses. In: Proceedings of the eighth REHVA world congress clima. Lausanne. Switzerland.

(27) Alonso, C., Oteiza, I., García-Navarro, J., Martín-Consuegra, F. (2016). Energy consumption to cool and heat experimental modules for theenergy refurbishment of fac, ades. Three case studies in Madrid. Energy and Buildings, 126, 252-262. doi: 10.1016/j.enbuild.2016.04.034

(28) Fernández-Agüera, J., Suárez R., Heiselberg, P. (2012). Influence of improvement of airtightness on energy retrofit of social Housing, a case study in a Mediterranean climate. 33rd AIVC Conference and 2nd TightVent Conference. Copenhague, Denmark 
(29) Giancola, E. Soutullo, S., Olmedo, R., Heras, M.R. (2014). Evaluating rehabilitation of the social housing envelope: experimental assessment of thermal indoor improvements duringactual operating conditions in dry hot climate, a case study. Energy and Buildings, 75, 264-271. doi: 10.1016/j.enbuild.2014.02.010

(30) Agencia Andaluza de la Energía. https://www.agenciaandaluzadelaenergia.es/es/noticias/el-programa-de-construccion-sostenible-aprueba-incentivar-con-158-millones-la-mejora-energetica-del-parque-publico-de-viviendas (acceso 2-12-2016) 